\title{
CUIDANDO DE QUEM CUIDA: AVALIANDO A QUALIDADE DE VIDA DE CUIDADORES DE AFÁSICOS
}

\author{
Taking care of the caregiver: valuing of the quality of life \\ of the aphasics caregivers
}

\author{
Ivone Panhoca ${ }^{(1)}$, Aline Cristina de Salles Pupo (2)
}

\begin{abstract}
RESUMO
Objetivo: avaliar a qualidade de vida de cuidadores de afásicos, utilizando-se o "Questionário de sobrecarga do cuidador (Burden Interview - Zarit)". Métodos: responderam as 22 questões do questionário, 30 cuidadores de afásicos, de ambos os sexos, que eram atendidos em uma clínica-escola de uma faculdade de Fonoaudiologia do interior do estado de São Paulo. Além disso, foram coletados os seguintes dados: sexo; idade; nível de escolaridade; há quanto tempo ocupava a posição de cuidador; grau de parentesco com o afásico; quais suas maiores dificuldades no trato com o afásico; tipo de afasia da pessoa sob seus cuidados (e acometimentos associados). Foram analisadas tais variáveis a fim de verificar como elas influenciavam na sobrecarga e, além disso, verificou-se a relação entre a pontuação geral do questionário e a questão em que o próprio cuidador respondia como avaliava sua sobrecarga como cuidador. Resultados: constatou-se que as variáveis "parentesco" e "dificuldades no trato com o afásico" estão relacionadas com a sobrecarga do cuidador, sendo que as maiores sobrecargas foram encontradas nos que cuidam de afásicos que apresentam maiores comprometimentos linguísticos e que se irritam facilmente quando não são compreendidos. Quanto ao grau de parentesco os pais de afásicos são os que se sentem mais sobrecarregados, seguidos pelos cônjuges, filhos e irmãos. Conclusão: os resultados encontrados mostraram que o cuidador do afásico apresenta sobrecarga física e emocional, sendo de extrema importância conhecer suas necessidades, para melhor assisti-los e orientá-los, buscando minimizar a sobrecarga e melhorando a qualidade de vida.
\end{abstract}

DESCRITORES: Saúde Pública; Qualidade de Vida; Acidente Cerebral Vascular; Afasia; Linguagem

\section{INTRODUÇÃO}

As sequelas advindas do acidente vascular cerebral (AVC) vão depender da extensão da lesão e da área atingida ', e uma das sequelas mais

(1) Fonoaudióloga; Docente da Faculdade de Fonoaudiologia da Pontifícia Universidade Católica de Campinas, UNICAMP, Campinas, SP, Brasil; Mestre e Doutora em Estudos da Linguagem pelo Instituto de Estudos da Linguagem da Universidade Estadual de Campinas; Pós-doutoramentos no Program in Communication Disorders da University of Houston/TX- USA, no Education Department da Washington University in St Louis/ MO-USA e na Facultad de Ciencias Sociales da Universidad de Salamanca, España.

(2) Fonoaudióloga; Bolsista de Iniciação Científica (Fundo de Apoio a Iniciação Científica-FAPIC/Reitoria da Pontifícia Universidade Católica de Campinas) no período agosto de 2007 - julho de 2008.

Conflito de interesses: inexistente comumente encontradas é a afasia, que se caracteriza por alterações de processos linguísticos de significação, que podem ser de origem articulatória e discursiva, produzidas por uma lesão adquirida no sistema nervoso central, em áreas responsáveis pela linguagem, podendo estar associadas a outras alterações do processo cognitivo ${ }^{2}$.

A afasia pode comprometer a compreensão e a expressão da linguagem, e mesmo não afetando necessariamente suas habilidades cognitivas e intelectuais pode levar o acometido ao isolamento e à depressão ${ }^{3}$. E os comprometimentos comunicativos, com repercussões nos aspectos psicossociais, trazem impactos também sobre a família do afásico ${ }^{4}$.

Os processos linguísticos são construídos nas relações sócio-culturais, destacando-se assim o papel do mediador, que organiza e estrutura a 
linguagem para o outro. $O$ fortalecimento da autoconfiança e auto-estima está relacionada ao modo como os sujeitos se reorganizam como intérpretes de um mundo, e isso se dá na relação com o outro, sendo que a qualidade das interações mantidas pelos afásicos fazem grande diferença na reabilitação linguísticas e na re-inserção social deles ${ }^{5}$.

Com a doença vascular cerebral podem ocorrer manifestações comportamentais associadas, sendo que muitas vezes o AVC ocorre no apogeu das capacidades criativas e profissionais do acometido, com os familiares se deparando com essa nova situação: o seu ente não será mais capaz de se comportar da maneira consequente, responsável e produtiva a que todos estavam acostumados pelos anos de convivência com o familiar ${ }^{6}$. E, além dos comprometimentos linguísticos, com frequência os sujeitos afásicos também passam a apresentar comprometimentos motores, que os tornam ainda mais dependentes de outra pessoa para o exercício de suas atividades de vida diária ${ }^{7}$.

Todas essas mudanças levam a pessoa acometida a necessitar de cuidados e os cuidadores, na sua grande maioria, passam a ocupar essa função sem a devida capacitação, o que tende a provocar desgaste tanto para a pessoa cuidada, quanto para o próprio cuidador ${ }^{8}$. Essa experiência de cuidar de um acometido no âmbito familiar vem se tornando cada vez mais comum surgindo, a partir dessa necessidade, o cuidador informal ${ }^{9}$, que costuma assumir esse papel ou por ter, com a pessoa sob cuidados, uma relação culturalmente definida, ou pelo vínculo afetivo que mantêm com ela ${ }^{10}$. Nesse cenário influenciam fortemente os fatores culturais, sendo que a família que não cumpre esse dever adequadamente passa a ser considerada negligente, vindo a sofrer sanções sociais ${ }^{11}$.

Cuidadores de pessoas que sofreram AVC vivenciam profundas mudanças no seu estilo de vida, ficando expostos a fatores limitantes da vida social e pessoal, tais como: a sobrecarga de atividades, perda do companheiro para as atividades sociais, enfrentamento de distúrbios de comportamentos da pessoa com AVC, mudanças nos relacionamentos familiares e de amizades. Apesar disso, com grande frequência observa-se relutância por suporte, com o cuidador mostrando-se resistente em aceitar auxílio ${ }^{12}$.

Além das mudanças na vida social com frequência os cuidadores passam a enfrentar limitações em sua vida profissional, que vão desde a redução da jornada de trabalho até o seu completo abandono, o que produz consequências como problemas financeiros, insatisfação por parte do cuidador e sentimento de culpa da pessoa acometida ${ }^{12,13}$. Portanto, a dedicação contínua ao familiar que necessita de cuidados interfere muito na vida dos cuidadores. Conforme o tempo passa, aumenta a demanda ${ }^{14} \mathrm{e}$ a sobrecarga, vivenciada por problemas ou dificuldades ${ }^{15}$ que comprometem o estado físico, psicológico e social do cuidador, causa prejuízo a qualidade de vida dele ${ }^{13}$.

Os comprometimentos da qualidade de vida (física e mental) do cuidador interferem de forma negativa no processo de reabilitação da pessoa cuidada ${ }^{16}$, uma vez que a sobrecarga testa os limites físicos, psicológicos, e a postura de enfrentamento do cuidador perante a vida ${ }^{17}$. Para que o cuidador não abandone o seu papel junto à pessoa acometida, mantendo-se saudável e com qualidade de vida, é de fundamental importância que ele aprenda a adaptar-se a essa nova condição ${ }^{18}$.

Considerada a importância de se conhecer a qualidade de vida de cuidadores de familiares dependentes, bem como os fatores intervenientes em tal tarefa, tem sido desenvolvidos estudos sobre a sobrecarga deles tanto por autores nacionais $10,12,16,19,20$ quanto por autores estrangeiros ${ }^{14,18,21-24}$. Na Fonoaudiologia, porém, ainda são poucos os trabalhos voltados aos cuidadores, conforme atestado pela literatura da área ${ }^{3,4}$.

O objetivo do estudo foi avaliar a qualidade de vida de cuidadores de afásicos, utilizando o Questionário de Sobrecarga do Cuidador Informal (“Burden Interview - Zarif”).

\section{MÉTODOS}

Trata-se de estudo observacional descritivo em que trinta cuidadores de afásicos, de ambos os sexos, na faixa etária de 15 a 75 anos, que eram atendidos em uma clínica-escola de fonoaudiologia de uma universidade particular do interior de São Paulo, foram convidados a responder às questões do "Questionário de Sobrecarga do Cuidador Informal" (QSCI), um instrumento de medidas genéricas utilizado internacionalmente para avaliar qualidade de vida de cuidadores, já validado no Brasil 25,26.

Foram incluídos na pesquisa cuidadores informais, ou seja, cuidadores que não recebiam nenhum tipo de remuneração, que detinham a maior responsabilidade pelos cuidados prestados ao afásico e que residiam ou passavam a maior parte de seu tempo com ele. Tais pessoas frequentavam o serviço de fonoaudiologia enfocado, na época da coleta de dados, e concordaram em assinar o Termo de Consentimento Livre e Esclarecido.

Foram excluídos desta pesquisa sujeitos que não se enquadravam no perfil de cuidador informal, descrito acima, e cuidadores que mesmo tendo o perfil se recusaram a participar da pesquisa. 
A versão brasileira do QSCI empregado nessa pesquisa é um instrumento usado como medida da sobrecarga do cuidador, sendo de grande confiabilidade e utilidade tanto em pesquisas como na prática clínica ${ }^{27}$.

O Questionário de Sobrecarga é um questionário, composto por 22 itens, que investiga a sobrecarga física, emocional e social em cuidadores informais. Há, ainda, uma questão em que o próprio cuidador pode avaliar o quanto se sente sobrecarregado por exercer a função de cuidador. Pode-se obter um score final que varia de 0 a 88 , sendo que, quanto maior a pontuação, maior será a sobrecarga apresentada pelo cuidador avaliado.

As variáveis analisadas em relação aos cuidadores foram: sexo; idade; nível de escolaridade; há quanto tempo ocupa a posição de cuidador; grau de parentesco com o afásico; quais suas maiores dificuldades no trato com o afásico; tipo de afasia da pessoa sob cuidados (e acometimentos associados), dados de extrema relevância ${ }^{28}$.

Este projeto foi aprovado pelo Comitê de Ética da Instituição em que se realizou o estudo (Protocolo 435/05).

Nessa pesquisa foram analisadas as variáveis dos cuidadores a fim de verificar como elas influenciavam na sobrecarga vivida por eles. Foi utilizada a técnica de regressão linear múltipla para investigar e modelar possíveis relacionamentos entre a sobrecarga do cuidador e suas características sócio-demográficas. Outro aspecto importante avaliado nesse estudo foi a relação entre a pontuação geral do questionário e a questão 'De maneira geral, quanto o $\mathrm{Sr} / \mathrm{Sra}$ se sente sobrecarregado(a) por cuidar de S?", questão em que o próprio cuidador deve responder como avalia, de forma geral, a sua sobrecarga. Para analisar o relacionamento entre a pontuação geral do questionário e essa questão específica, foi computado o coeficiente de correlação de Spearman.

\section{RESULTADOS}

Entre os 30 cuidadores enfocados nesse estudo, foram encontradas 24 mulheres ( $80 \%)$, sendo que dentre essas se encontram 14 esposas de acometidos $(46,67 \%)$, seguidas por sete filhas $(23,33 \%)$, duas mães $(6,67 \%)$ e uma irmã $(3,33 \%)$. Homens responderam por $20 \%$ do total dos cuidadores, sendo que três deles (10\%) eram esposos de acometidas, seguidos por dois irmãos $(6,67 \%)$ e um pai $(3,33 \%)$. Não foram encontrados filhos na posição de cuidadores.

Os entrevistados tinham entre 15 e 75 anos, sendo 12 deles (40\%) com mais de 60 anos de idade, verificando-se, então grande parte dos cuidadores é idoso, cuidando de idosos.

Quanto ao tempo de cuidado com o afásico, 15 $(50 \%)$ prestavam cuidados por um período inferior a dois anos, nove cuidadores (30\%) de dois anos e meio a cinco anos, três cuidadores (10\%) de cinco a 10 anos e três desses cuidadores (10\%), há mais de 10 anos.

Em relação ao nível de escolaridade dos cuidadores prevaleceram os que possuíam ensino fundamental incompleto, sendo que nenhum cuidador enfocado tinha nível superior.

A maioria dos cuidadores, 13 familiares $(43,33 \%)$, relatou que sua maior dificuldade com o afásico era entender sua linguagem, seguido por problemas com atividades de vida diária e por dificuldades com os aspectos emocionais e motores. Somente dois cuidadores $(6,66 \%)$ relataram não ter nenhum tipo de dificuldade no trato com o familiar, sendo importante destacar que 10 cuidadores $(33,33 \%)$ relataram ter mais de uma dificuldade no trato com o afásico (Tabela 1).

Os cuidadores enfocados nesse estudo tinham sob seus cuidados 17 afásicos não fluentes (56,6\%) e 13 afásicos fluentes (43,4\%). Desse total de afásicos, $13(43,4 \%)$ apresentavam marcha independente, seguidos de nove afásicos $(30 \%)$ com marcha semi-independente e oito cadeirantes $(26,6 \%)$. Como observado na Tabela 2, a média de sobrecarga dos cuidadores, quanto ao tipo de afasia e marcha do familiar sob cuidados, se mantém entre 25 e 27 pontos (Tabela 2).

Cabe ressaltar que a pontuação dos indivíduos desconsiderando qualquer categoria referente a variáveis demográficas apresentou um valor mínimo de zero e o valor máximo foi de 51 pontos. O primeiro quartil foi aproximadamente 14, o que significa que $25 \%$ dos entrevistados obtiveram pontuação entre zero e 14. Da mesma forma, o terceiro quartil foi 33, indicando que $25 \%$ dos indivíduos obtiveram pontuação entre 33 e 51 pontos. Consequentemente, verificou-se que $50 \%$ dos cuidadores tiveram pontuação entre 15 e 32 pontos. Lembrando que a pontuação geral do questionário varia de 0 a 88 pontos, observou-se aqui, então, uma predominância de valores relativamente baixos considerando-se a pontuação máxima do questionário.

Depois de se ajustar um modelo de regressão linear múltiplo, foi constatado que as co-variáveis "parentesco" e "dificuldades no trato com o afásico" influenciavam significativamente na sobrecarga dos cuidadores, sendo que quando passa-se das categorias de Irmão(ã) para Filho, desta para Marido/ Esposa e desta para Mãe/Pai, há um aumento médio esperado de aproximadamente 5,6 pontos na pontuação total de sobrecarga dos cuidadores. 
Tabela 1 - Proporção de participantes do estudo consideradas as variáveis demográficas

\begin{tabular}{|c|c|c|c|}
\hline Categoria & Status & $\mathrm{N}^{\circ}$ participantes & Porcentagem \\
\hline \multirow{2}{*}{ Sexo } & Feminino & 24 & $80,0 \%$ \\
\hline & Masculino & 6 & $20,0 \%$ \\
\hline \multirow{4}{*}{ Parentesco } & Irmão(a) & 3 & $10,0 \%$ \\
\hline & Filho(a) & 7 & $23,4 \%$ \\
\hline & Marido/Esposa & 17 & $56,6 \%$ \\
\hline & Mãe/Pai & 3 & $10,0 \%$ \\
\hline \multirow{4}{*}{ Faixa Etária } & $15-30$ & 3 & $10,0 \%$ \\
\hline & $31-45$ & 3 & $10,0 \%$ \\
\hline & $46-60$ & 12 & $40,0 \%$ \\
\hline & $>60$ & 12 & $40,0 \%$ \\
\hline \multirow{4}{*}{ Tempo como cuidador } & $<2$ anos e $1 / 2$ & 15 & $50,0 \%$ \\
\hline & $2,5-5$ anos & 9 & $30,0 \%$ \\
\hline & 5-10 anos & 3 & $10,0 \%$ \\
\hline & $>10$ anos & 3 & $10,0 \%$ \\
\hline \multirow{5}{*}{ Escolaridade } & Analfabeto & 2 & $6,6 \%$ \\
\hline & Fundamental Inc & 17 & $56,6 \%$ \\
\hline & Fundamental Comp & 4 & $13,4 \%$ \\
\hline & Médio Inc & 4 & $13,4 \%$ \\
\hline & Médio Comp & 3 & $10,0 \%$ \\
\hline \multirow{4}{*}{ Dific. no trato } & Linguagem & 13 & $43,33 \%$ \\
\hline & Aspecto Motor & 5 & $16,60 \%$ \\
\hline & Ativ. vida diária & 11 & $36,66 \%$ \\
\hline & Aspectos emocionais & 9 & $30,0 \%$ \\
\hline \multirow{3}{*}{$\mathrm{N}^{\circ}$ de dificuldades } & Nenhuma & 2 & $6,66 \%$ \\
\hline & Uma & 18 & $60,00 \%$ \\
\hline & Duas & 10 & $33,33 \%$ \\
\hline
\end{tabular}

Técnica de regressão linear múltipla

Tabela 2 - Estatísticas descritivas consideradas: tipo de afasia e marcha do afásico sob cuidados

\begin{tabular}{lccc}
\hline Categoria & Status & Média & Desvio Padrão \\
\hline \multirow{2}{*}{ Fluência } & Não & 25,53 & 12,50 \\
& Sim & 24,92 & 12,76 \\
\hline \multirow{2}{*}{ Marcha } & Independente & 23,54 & 13,03 \\
& Semi-independ. & 25,67 & 14,20 \\
& Cadeirante & 27,63 & 10,16 \\
\hline
\end{tabular}

Os cuidadores que relataram ter dificuldades para entender a linguagem do afásico têm um aumento médio esperado de aproximadamente 8,5 pontos na sua sobrecarga em relação àqueles que relataram não ter nenhuma dificuldade no trato com o afásico. Os que relataram ter dificuldades com os aspectos emocionais do afásico tiveram aumento médio de aproximadamente 10,1 pontos de sobrecarga na pontuação geral.
Embora na análise descritiva tenham sido encontradas algumas diferenças nas médias das pontuações para outras co-variáveis, estatisticamente elas não são significativas para alterações esperadas na resposta.

Quando os cuidadores foram questionados sobre o quanto se sentiam sobrecarregados na posição que ocupavam nove deles $(30 \%)$ relataram não sentir nenhuma sobrecarga, 12 cuidadores (40\%) 
Tabela 3 - Estatísticas descritivas consideradas as variáveis demográficas

\begin{tabular}{lccc}
\hline Categoria & Status & Média & Desvio Padrão \\
\hline \multirow{2}{*}{ Sexo } & Feminino & 26,58 & 11,41 \\
& Masculino & 20,00 & 15,84 \\
\hline \multirow{3}{*}{ Parentesco } & Irmão(a) & 12,00 & 13,11 \\
& Filho(a) & 23,29 & 9,20 \\
& Marido/Esposa & 27,29 & 11,00 \\
Faixa Etária & Mãe/Pai & 31,7 & 21,5 \\
& $15-30$ & 34,0 & 17,0 \\
& $31-45$ & 20,0 & 11,53 \\
& $46-60$ & 25,58 & 12,87 \\
Tempo como cuidador & $>60$ & 24,08 & 11,52 \\
\hline \multirow{4}{*}{ Escolaridade } & 2 anos e $1 / 2$ & 23,53 & 13,49 \\
& $2,5-5$ anos & 26,22 & 9,34 \\
& $5-10$ anos & 24,67 & 7,51 \\
& $>10$ anos & 31,7 & 21,5 \\
\hline No de dificuldades & Analfabeto & 35,5 & 14,8 \\
& Fundamental Inc & 22,41 & 12,12 \\
& Fundamental Comp & 20,00 & 8,98 \\
& Médio Inc & 35,25 & 14,43 \\
& Médio Comp & 28,33 & 8,14 \\
\hline & Nenhuma & 5,00 & 7,07 \\
Dificuldades no trato & Linguagem & 28,92 & 11,48 \\
& Aspecto Motor & 25,40 & 12,36 \\
& Ativ. vida diária & 23,45 & 11,45 \\
& Aspectos emocionais & 32,22 & 10,50 \\
\hline & Nenhuma & 5,00 & 7,07 \\
& Uma & 24,72 & 11,20 \\
& Duas & 30,30 & 11,51 \\
\hline
\end{tabular}

Técnica de regressão linear múltipla

um pouco, seis cuidadores (20\%) moderadamente, dois cuidadores $(6,7 \%)$ muito e um cuidador $(3,3 \%)$ relatou sentir-se extremamente sobrecarregado.

$\mathrm{Na}$ questão "De maneira geral, quanto o $\mathrm{Sr} /$ Sra se sente sobrecarregado(a) por cuidar de S?", o cuidador deve responder como ele avalia, de forma geral, a sua sobrecarga como cuidador. Relacionando-se a pontuação de sobrecarga geral dos cuidadores com as respostas que esses deram a essa questão (e a fim de se avaliar o relacionamento entre essas duas variáveis) foi computado o coeficiente de correlação de Spearman.

Utilizando o coeficiente Spearman verificou-se, então, que quando o valor relatado na questão enfocada aumentava, a pontuação geral do questionário do cuidador também tendia a ser maior observando-se, então, uma relação positiva entre essas variáveis.
Como se observa na Figura 1, quanto mais próximos da reta (pontilhada) estiverem os pontos, maior será o valor absoluto do coeficiente de correlação e a relação positiva entre as variáveis. Porém, o coeficiente de correlação Spearman não teve um valor considerado alto, fato este que pode ser explicado pela presença de subjetividade nas respostas, ou ainda por alguns cuidadores não admitirem sentirse sobrecarregado na tarefa de cuidar.

\section{DISCUSSÃO}

Em concordância com estudos da área 9-11, 13-15,17,20,22,25-28 os resultados encontrados apontam uma prevalência de pessoas do sexo feminino na posição de cuidadores, o que pode ser explicado pelo papel histórico e cultural que é atribuído à mulher, que no passado restringia-se a cuidar das atividades domésticas, do marido 


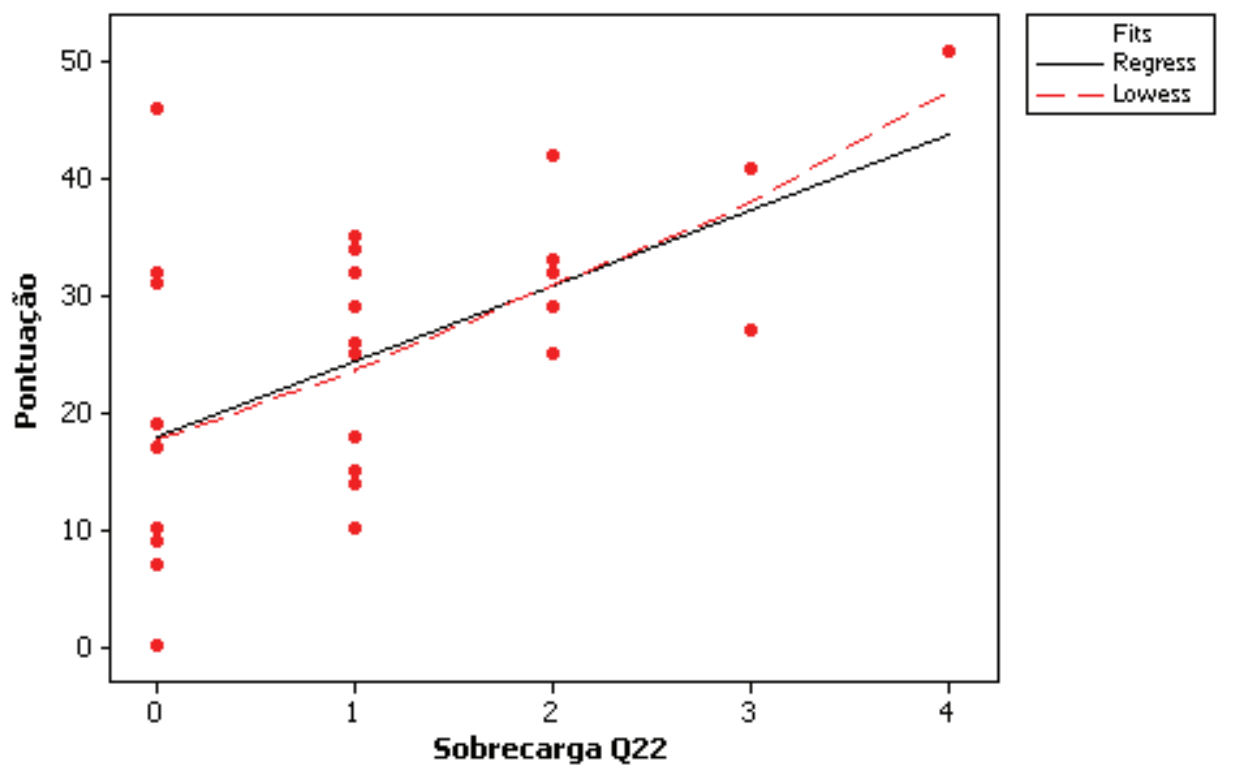

Figura 1 - Diagrama de Dispersão: Pontuação versus Sobrecarga

e dos filhos, incumbida de tudo que se relacionasse ao cuidar do outro ${ }^{10,11,28}$.

A faixa etária da maior parte dos cuidadores $(80 \%)$ foi superior a 45 anos, o que pode estar relacionado com a prevalência dos casos de AVC em idades mais avançadas, como lembram alguns autores que relatam que o AVC está entre as quatro primeiras causas de morte em adultos e que ele ocorre com maior frequência à medida que a população envelhece. Com a prevalência de cônjuges como cuidadores, justifica-se a prevalência de cuidadores idosos, em conformidade com o encontrado em outro estudo da área ${ }^{1}$. Essa prevalência de cônjuges na posição de cuidadores pode ser vista, como já foi dito aqui, como um dever embutido no compromisso do matrimônio, em conformidade com a nossa cultura, mas deve-se considerar, também, que a afetividade pode estar contribuindo para que esse cônjuge cuide do outro, uma vez que esse componente afetivo é um fator que influencia na escolha de tal função ${ }^{10}$.

O tempo de cuidado prestado ao afásico, na maioria dos casos (50\%), foi inferior a 2 anos, um tempo relativamente baixo perante os demais cuidadores que assumiram tal tarefa há mais de 2 anos e meio ou até mesmo há mais de 10 anos.

Para a população do presente estudo a variável tempo como cuidador não influenciou na sobrecarga dos cuidadores, em discordância com estudos da área que mostraram que conforme o tempo passa aumenta a demanda e a sobrecarga do cuidador ${ }^{14,15,20,22}$.

O nível de escolaridade dos cuidadores enfocados, em sua maioria, foi de ensino fundamental incompleto, lembrando tratar-se de pessoas atendidas em serviço fonoaudiológico gratuito, voltado à população de baixa renda.

A literatura da área destaca que o cuidador quase sempre sente que não tem outra opção que não seja a de cuidar, resignando-se nessa posição, e no presente estudo verificou-se que grande parte dos cuidadores passaram a ocupar essa posição por não haver outra pessoa que pudesse fazê-lo ${ }^{11}$.

Em muitos casos, portanto, não se verifica o "cuidado por afinidade", que alguns autores destacam como sendo de grande importância uma vez que a ausência dessa "afinidade" pode comprometer a assistência dada ${ }^{8}$.

No presente estudo não foi encontrada influência das variáveis sexo, idade, tempo na posição de cuidador e grau de escolaridade na sobrecarga dos cuidadores. Da mesma forma, o tipo de afasia (fluente ou não fluente) e a marcha (independente, semi-independente e cadeirante), não influenciaram na sobrecarga do cuidador.

Por meio de técnicas de análises de regressão constatou-se que apenas as variáveis "parentesco" e "dificuldades no trato com o afásico" estão relacionadas com a sobrecarga do cuidador, verificandose que as maiores sobrecargas são encontradas nos que cuidam de afásicos que apresentem dificuldades com a linguagem e que se irritam facilmente quando não são compreendidos.

Quanto ao parentesco, constatou-se que pais de afásicos são os que se sentem mais sobrecarregados, seguidos pelos cônjuges, filhos e os menos sobrecarregados são irmãos dos acometidos. Esses altos níveis de sobrecarga podem estar associados 
à constatação, pelo cuidador, de que sua estrutura familiar está sendo afetada por uma modificação de papéis sociais. A mãe que cuidou de seus filhos, por exemplo, esperava que quando envelhecesse e necessitasse de cuidados eles poderiam ser prestados pelos filhos. Ao se deparar com uma situação inversa, em que o filho, ainda jovem, sofre um AVC e necessita de cuidados, ela toma para si esse papel, porém diante de uma situação que não considera como natural ${ }^{17}$.

A esposa, que até o acometimento do marido, se dedicava à casa e à família, foi impelida a assumir a responsabilidade do orçamento doméstico e das decisões importantes para aquela família ${ }^{9}$, fato este em consonância com dados do presente estudo, que mostraram que cônjuges, em sua maioria mulheres, representam a segunda categoria mais sobrecarregada no que diz respeito ao grau de parentesco.

Também em consonância com os dados do presente estudo, outro autor verificou que a variável grau de parentesco influenciou no nível de sobrecarga do cuidador, sendo que em seu estudo mãe e filhas apresentaram as maiores pontuações no questionário de sobrecarga ${ }^{20}$.

No que diz respeito ao trato com a pessoa sob cuidados, as maiores sobrecargas foram as dos cuidadores que tinham dificuldade de entender a linguagem do afásico, dado já destacado por outras autoras que destacaram as dificuldades de comunicação (seja de expressão, seja de compreensão) como fatores que influenciaram a relação do afásico com seus familiares ${ }^{4}$.

Também em consonância com essas autoras ${ }^{4}$ verificou-se que os cuidadores dão destaque para a irritação dos afásicos quando não são compreendidos e para a forma como isso os afeta, tendo-se encontrado um aumento esperado de 10,1 pontos na pontuação geral do questionário em cuidadores que relataram esse tipo de dificuldade com o afásico.

Tal dado nos mostra a importância da intervenção dos profissionais da área da saúde, em especial do fonoaudiólogo, no que diz respeito à melhoria da qualidade da interação linguística entre cuidador e afásico ${ }^{5,29}$.

Os níveis prevalentes de sobrecarga dos cuidadores desse estudo estiveram entre 15 e 32 pontos $(50 \%)$, um score relativamente baixo comparado com a pontuação máxima do questionário (88 pontos). Entretanto, $25 \%$ dos cuidadores mostraram sobrecarga significativa, com pontuações entre 33 e 51 pontos. Tais dados vão ao encontro dos observados por outros dois estudos, em que o primeiro encontrou uma média de 32 postos de sobrecarga nos cuidadores pesquisados ${ }^{15}$ e no segundo estudo a pontuação dos cuidadores permaneceu com médias entre 23 e 30 pontos ${ }^{26}$.

A sobrecarga vivenciada pelo cuidador, desencadeada pelos efeitos negativos das incapacidades geradas pelo AVC, pode interferir no cuidado prestado ao acometido ${ }^{15}$ e por esse motivo é necessário que o cuidador conheça a sua sobrecarga e consiga compartilhá-la, criando estratégias que busquem melhorar sua qualidade de vida, sendo amparado no âmbito familiar e profissional, tornando-se imprescindível as políticas públicas que promovam programas de avaliação e intervenção continuada nas equipes de saúde ${ }^{12,19}$.

Todos os cuidadores participantes dessa pesquisa eram familiares próximos da pessoa afásica, que cumpriam função sócio-econômica de servir de suporte e solução dos problemas da pessoa acometida ${ }^{18}$.

O papel de cuidador, atribuído por questões religiosas e culturais, pode ajudar no enfrentamento do cuidar, trazendo ao cuidador satisfação pelo desempenho de tal tarefa, perante si próprio e perante a sociedade. A pessoa sob cuidados, por sua vez, pode se sentir amparada e encorajada no enfrentamento da doença ${ }^{23}$.

O apoio do grupo social a que o cuidador pertence pode ajudar na diminuição da carga sentida por ele, melhorando sua qualidade de vida, e consequentemente a do familiar que necessita de cuidados ${ }^{16,22}$.

As doenças físicas podem assumir uma variedade de formas, mas é no âmbito familiar que elas vêm sendo resolvidas aqui no Brasil ${ }^{19}$. Por essa razão é que os profissionais da equipe de saúde devem fornecer o treinamento e o suporte que os familiares necessitam para exercer o cuidado domiciliar ${ }^{7,19,21}$, visando à diminuição das hospitalizações e a redução de custos e auxiliando na reinserção social e na melhora da qualidade de vida do acometido e de sua família. E ao fonoaudiólogo, especificamente, cabe favorecer a recuperação da linguagem e minimizar as sequelas lingüísticocomunicativas do $\mathrm{AVC}^{3}$.

Outro aspecto que deve ser lembrado é a importância do cuidador aprender a cuidar de si, recebendo ajuda emocional e física ${ }^{24}$. Ele deve aprender a prestar a atenção à pessoa dependente, sem esquecer que ele também precisa se cuidar, inclusive porque para cuidar bem de outra pessoa é necessário antes de tudo, saber cuidar de si. 


\section{CONCLUSÃO}

Os resultados encontrados mostraram que 0 cuidador do afásico apresenta sobrecarga física e emocional necessitando, portanto, do acolhimento e da atenção do fonoaudiólogo, tanto quanto o próprio afásico.
Importante destacar que grande número de cuidadores referiu que sua maior dificuldade no trato com o afásico era entender a linguagem dele, sendo que esse aspecto foi significativo no aumento da sobrecarga de tais cuidadores, o que mostra a importância da intervenção fonoaudiológica junto a essa população.

\begin{abstract}
Purpose: to value aphasics' caregivers quality of life using the "Caregiver Burden Questionnaire (Burden Interview - Zarit)". Methods: thirty aphasics' caregivers, of both genders, answered twentytwo questions of the questionnaire, and were attended in a clinic-school at a Speech Therapy University located in the western part of São Paulo state. Besides, the following details were collected: gender; age; school education; time working as caregivers; degree of relationship aphasic-caregiver; difficulties faced during the aphasics' treatment; the type of aphasia that the person under their cares has (and associated attacks). Such variables were analyzed in order to check how they were influencing the burden and, in addition to the relation between the general score of the questionnaire and the question answered by the caregivers valuing their own burden. Results: we found that the variables "relationship" and "difficulties faced during the aphasic treatment" are related to the caregiver's burden, although the higher burdens were found in caregivers who take care of aphasics showing severe linguistic compromising and become easily irritated when they are not understood. In terms of relationship, aphasic's parents are those who feel more burdens, followed by the spouses, children and brothers. Conclusion: the results showed that the aphasic's caregivers shows physical and emotional burden, being of extreme importance knowing their necessities, to better assist and guide them, trying to minimize the burden and improving quality of life.
\end{abstract}

KEYWORDS: Public Health; Quality of Life; Stroke; Aphasia; Language

\section{REFERÊNCIAS}

1. Quagliato EMAB, Viana MA. Orientações aos cuidadores de pacientes com doenças neurológicas crônicas. In: Dias ELF, Wanderley JS, Mendes RT. Orientações para cuidadores informais na assistência domiciliar. Campinas: UNICAMP; 2002. p. 119-36.

2. Coudry MIH. Diário de Narciso: discurso e afasia: análise discursiva de interlocuções com afásicos. São Paulo: Martins Fontes; 2001.

3. Belleza AMDO, Calegari VS, Raggio APR, Andrade GHM. Atuação fonoaudiológica em parceria com o "Programa Médico da Família" junto ao paciente com acidente vascular encefálico. Rev. CEFAC. 2003; 5(1):31-9.

4. Michelini CRS, Caldana ML. Grupo de orientação fonoaudiológica aos familiares de lesionados cerebrais adultos. Rev. CEFAC. 2005; 7(2):137-48.

5. Morato EM. Rotinas significativas e práticas discursivas: relato de experiência de um centro de convivência de afásicos. Rev Dist Comun. 1999; 10(2):157-65.

6. Guimarães dos Santos CLN. Aspectos comportamentais em doenças crônicas: terapeuta, cuidador e paciente. In: Levy JA, Oliveira ASB. Reabilitação em doenças neurológicas: guia terapêutico prático. São Paulo: Atheneu; 2003. p. 223-9.

7. Lavinsky AE, Vieira TT. Processo de cuidar de idosos com acidente vascular encefálico: sentimentos dos familiares envolvidos. Acta Sci Health Sci. 2004; 26(1):41-5.

8. Saliba NA, Moimaz SAS, Marques JA, Marques JAM, Prado RL. Perfil de cuidadores de idosos e percepção sobre saúde bucal. Interface Comun Saúde Educ. 2007; 11(21):39-50.

9. Perlini NMOG, Faro ACM. Cuidar de pessoa incapacitada por acidente vascular cerebral no domicílio: o fazer do cuidador familiar. Rev Esc Enferm USP. 2005; 39(2):154-63.

10. Cattani RB, Girardon-Perlini NMO. Cuidar do idoso doente no domicílio na voz de cuidadores familiares. Rev Eletr Enf. Mai-Ago 2004; 6(2):254-71. 
11. Caldas CP. O idoso em processo de demência: o impacto na família. In: Minayo MCS, Coimbra JR, Carlos EA. Antropologia, saúde e envelhecimento. Rio de Janeiro: Fiocruz; 2002. p. 51-71.

12. Bocchi SCM. Vivenciando a sobrecarga ao vira-ser um cuidador familiar de pessoa com acidente vascular cerebral (AVC): análise do conhecimento. Rev Latino-Am Enferm 2004; 12(1):115-21.

13. Gonçalves LHT, Alvarez AM, Sena ELS, Santana LWS, Vicente FR. Perfil da família cuidadora de idoso doente/fragilizado do contexto sociocultural de Florianópolis, SC. Texto Contexto Enferm. 2006; 15(4):570-7.

14. Herrera SEMC. Calidad de vida de los cuidadores familiares que cuidan niños en situación de enfermedad crónica. Av Enferm. 2004; 22(1):39-46. 15. Cassis SVA, Karnakis T, Moraes TA, Curiati JAE, Quadrante ACR, Magaldi RM. Correlação entre o estresse do cuidador e as características clínicas do paciente portador de demência. Rev Assoc Med Bras. 2007; 53(6):497-501.

16. Bocchi SCM, Ângelo M. Entre la libertad y la reclusión: el apoyo social como un componente de la calidad de vida del binomio cuidador familiar y persona dependiente. Rev Latino Am Enferm. 2008; 16(1):15-23.

17. Luzardo AR, Gorini MIPC, Silva APSS. Caracteristicas de idosos com doença de Alzheimer e seus cuidadores: uma série de casos em um Serviço de Neurogeriatria. Texto Contexto Enferm. 2006; 15(4):587-94.

18. Gómez MMG. Estar ahí, al cuidado de un paciente con demencia. Invest Educ Enferm. 2007; 25(2):60-71.

19. Bocchi SCM, Ângelo M. Interação cuidador familiar - pessoa com AVC: autonomia compartilhada. Ciênc Saúde Coletiva. 2005; 10(3):729-38.

20. Garrido R, Menezes PR. Impacto em cuidadores de idosos com demência atendidos em um serviço psicogeriátrico. Rev Saúde Pública. 2004; 38(6):835-41.
21. Bookman A, Harrington M. Family caregivers: a shadow workforce in the geriatric health care system?. J Health Polit Policy Law. 2007 Dec; 32(6):1005-41.

22. Turro-Garriga O, Soler-Cors O, Garre-Olmo J, Lopez-Pousa S, Vilalta-Franch J, Monserrat-Vila S. Distribución factorial de la carga en cuidadores de pacientes con enfermedad de Alzheimer. Rev Neurol. 2008; 46(10):582-8.

23. Jullamate $P$, de Azeredo Z, Rosenberg E, Pàul C, Subgranon R. Informal stroke rehabilitation: what are the main reasons of Thai caregivers? Int $\mathrm{J}$ Rehabil Res. 2007 Dec; 30(4):315-20.

24. Steiner V, Pierce L, Drahuschak S, Nofziger E, Buchman D, Szirony T. Emotional support, physical help, and health of caregivers of stroke survivors. J Neurosci Nurs. 2008 Feb; 40(1):48-54.

25. Martins T, Ribeiro JP, Garret C. Estudo de validação do questionário de avaliação da sobrecarga para cuidadores informais. Psicol Saúde Doenças. 2003; 4(1):131-48.

26. Westphal AC, Alonso NB, Silva TI, Azevedo AM, Caboclo LOSF, Garzon E, et al. Comparação da qualidade de vida e sobrecarga dos cuidadores de pacientes com epilepsia por esclerose mesial temporal e epilepsia mioclônica juvenil. J Epilepsy Clin Neurophysiol. 2005 Jun; 11(2):71-6

27. Taub A, Andreoli SB, Bertolucci PH. Dementia caregiver burden: reliability of the Brazilian version of the Zarit caregiver burden interview. Cad Saúde Pública. 2004 Mar/Apr; 20(2):372-6.

28. Mendes PBMT. Quem é o cuidador. In: Dias ELF, Wanderley JS, Mendes RT. Orientações para cuidadores informais na assistência domiciliar. Campinas: UNICAMP; 2002. p. 19-33.

29. Morato EM. Sobre as afasias e os afásicos: subsídios teóricos e práticos elaborados pelo Centro de Convivência de Afásicos. Campinas: UNICAMP; 2002.

RECEBIDO EM: 14/08/2008

ACEITO EM: 10/11/2009

Endereço para correspondência:

Ivone Panhoca

Estrada da Rhodia, 5150 casa 28

Campinas - SP

CEP: $13085-850$

E-mail: i.panhoca@terra.com.br 